\section{Intersections}

Canadian Journal of Music

Revue canadienne de musique
Intersections CANADIAN JOURAL OF MUSIC

\title{
Un entretien avec Diane Labrosse
}

\section{Andrea-Jane Cornell}

Volume 26, numéro 2, 2006

In and Out of the Sound Studio

URI : https://id.erudit.org/iderudit/1013227ar

DOI : https://doi.org/10.7202/1013227ar

Aller au sommaire du numéro

\section{Éditeur(s)}

Canadian University Music Society / Société de musique des universités canadiennes

\section{ISSN}

1911-0146 (imprimé)

1918-512X (numérique)

Découvrir la revue

Citer ce document

Cornell, A.-J. (2006). Un entretien avec Diane Labrosse. Intersections, 26(2), 75-80. https://doi.org/10.7202/1013227ar

\section{Résumé de l'article}

Cet entretien avec Diane Labrosse a été réalisé à Montréal en décembre 2004 dans les locaux de Productions SuperMusique, dans le cadre du projet Dedans/Dehors du studio coordonné par Dre Andra McCartney de l'Université Concordia.

Labrosse se définit comme musicienne, même si elle considère que ce titre ne couvre pas vraiment l'ensemble des rôles assumés dans divers ensembles et projets depuis 25 ans quelle exerce ce métier. L'essentiel de son activité est à titre de conceptrice sonore, en particulier dans les projets pour le théâtre, les concerts " live " et les performances de musique improvisée avec d'autres musiciens, danseurs ou acteurs de théâtre. Avec son instrument de prédilection, un échantillonneur, Diane crée des ambiances sonores composées de sons inhabituels, de bruits et de textures, avec un certain penchant pour des modifications brusques de hauteurs.
Copyright (C Canadian University Music Society / Société de musique des universités canadiennes, 2007
Ce document est protégé par la loi sur le droit d'auteur. L’utilisation des services d’Érudit (y compris la reproduction) est assujettie à sa politique d'utilisation que vous pouvez consulter en ligne.

https://apropos.erudit.org/fr/usagers/politique-dutilisation/ 


\section{Un eNTRETIEN AVEC DiANe LABRosse}

\section{Andrea-Jane Cornell}

L’entretien avec Diane Labrosse a été réalisé à Montréal en décembre 2004 dans les locaux de Productions SuperMusique, dans le cadre du projet Dedans/Dehors du studio coordonné par Dre Andra McCartney de l'Université Concordia.

Diane Labrosse est une musicienne active sur les scènes de musiques progressives, actuelles et expérimentales, et s'est imposée comme compositeur et interprète depuis plus de 25 ans. Elle est un membre fondateur de Wondeur Brass, Justine et Les Poules, avec Diane Pallardy Roger et Joane Hétu. Elle collabore actuellement avec des gens de la scène locale et internationale. Pour le moment, elle collabore avec Martin Tétreault sur le projet Parasites, le trio Île bizarre également avec Tétreault et Ikue Mori, et Morceaux de Machines avec Aimé Dontigny, pour nen nommer que quelques-uns. Elle applique également ses univers sonores improvisés aux univers de la danse et du théâtre. Sa discographie contient plus de 40 parutions. Elle est co-directrice du groupe Productions SuperMusique, maison de promotion et organisation de spectacles de musiques innovatrices et actuelles.

\section{Comment vous définissez-vous en tant que créatrice?}

La plupart du temps je me définis comme musicienne, même si ce nest pas exactement mon rôle dans un projet. C'est plutôt comme être une conceptrice sonore, en particulier dans les projets pour le théâtre. C'est plus de la conception sonore, mais jai tendance à me définir comme une musicienne. Je fais beaucoup de concerts " live » et de performances de musique improvisée. Même si je travaille avec des instruments électroniques, je fais tout de même plus de concerts que de musique en studio et je pense que c'est d'ailleurs ça qui mamène toujours à me définir comme une musicienne.

\section{Depuis combien de temps travaillez-vous comme musicienne?}

Oh boy.... Ça fait longtemps! Ça fait à peu près 25 ans ou un peu plus que je fais de la musique.

Quelle est la première expérience sonore qui vous ait marquée quand vous étiez jeune? Ou la première expérience assez formidable pour sortir de l'ordinaire?

Diane éprouvait de la difficulté à se rappeler sa première expérience sonore. Elle décrit sa famille comme nétant pas particulièrement artistique ou musicale. Ce n'est que lorsqu'elle apprendra le piano, qu'elle s'engagera dans une relation par- 
ticulière avec le son. Son parcours musical a débuté avec de la musique traditionnelle en utilisant la voix, le piano et l'accordéon, en passant par la composition de musique pour orchestre ou petit ensemble, dérivant vers le rock progressif. Ensuite, elle en est venue à l'improvisation et, parallèlement d̀ cela, elle est passée du piano électrique au synthétiseur pour finalement en arriver à l'échantillonneur quelle continue à utiliser comme instrument de choix aujourd'hui.

Il y a environ 10 ans, jai décidé de travailler à partir de bruits échantillonnés du quotidien. Je voulais essayer d'en faire une musique, peut-être même des chansons ou quelque chose d'assez mélodieux, d'organisé. Ce fût peut-être l'expérience sonore qui ma le plus transformé au niveau de lécoute, même si c'était un peu tardif dans ma vie. C'est à ce moment que j'ai vraiment commencé à écouter.

Cest par le montage dune banque sonore que Diane prendra conscience du bruit, qui depuis ce temps sera d la base de ses cuvres musicales.

Vous me disiez que vous étiez plutôt " self taught " (autodidacte). Pourriezvous décrire un peu votre formation?

Je suis complètement " self-taught " (autodidacte), à part les cours de piano qui mont initié à la musique (j’ai appris à lire et à écrire la musique et tout le reste...). Bon d'accord... parfois je saute quelques étapes en consultant des gens qui sont vraiment bons dans le domaine. Ils me disent " regarde, c'est de même que tu le fais ". Ou parfois, quand je suis avec quelqu'un d'autre, j’en profite pour lui montrer comment moi je fais. C'est un échange intéressant.

Ce n'est qu'après quelques années que Diane acceptera (ou assumera complètement) son titre d'autodidacte: sa formation n'est pas académique, certes, mais elle est tout aussi valable.

Si vous avez à décrire le son, est-ce que vous avez tendance à vous orienter vers une description technique, une ambiance, une émotion ou le message que le son contient? Et comment ça te fait ressentir?

Le son, pour moi, c'est rarement technique, je ne peux pas dire à quelqu'un : « baisse ça autour de trois cents ". Ce n'est pas au niveau technique, mais c'est beaucoup plus au niveau des émotions. Ce qu'une sorte de son ou un ensemble de son peut provoquer comme émotion, cela m'intéresse beaucoup et même quand je suis dans l'abstraction totale, parce que c'est vraiment juste des "scrountch" ou des "buzz» il y a des moments où il y a des émotions qui sont vraiment dégagées de ça, qui ressortent de ça et qui viennent me toucher super fort. Des fois, après une session d'enregistrement ou en écoutant un enregistrement d'un concert, il arrive un passage chargé dẻmotions et souvent ce ne sont pas les passages qui sont les plus chargés de sons, mais c'est justement l'absence de son ou la retenue de chaque personne ou encore la place quon a laissé au silence qui fait qu'il y a vraiment une émotion qui se dégage d'un passage et c'est ça que jaime le plus.

\section{Comment décririez-vous votre travail aujourd'hui?}

Je suis vraiment intéressée par des textures. Peut-être que j’emploie ce mot un petit peu librement, mais jaime beaucoup mieux avoir des sons que je peux classer comme si on pouvait les toucher. Des choses qui sont bien granuleu- 
ses ou qui sont comme des petits chocs électriques: c'est tout un univers qui m'intéresse. Je dirais que je travaille maintenant beaucoup avec ces textures. En concert d'improvisation complète, sans parcours, j'aime travailler avec le moment présent, lécoute et puis la réponse. Puis avec d’autres personnes, il y a évidemment un jeu qui s'installe entre deux personnes en improvisation.... Ce n'est jamais pareil et ne pourra jamais lêtre, de toute façon. C'est un jeu qui te demande dềtre complètement réceptif à ce que l'autre propose.

Diane explique quen improvisation, elle a le choix de suivre le chemin quion lui propose ou de s'en écarter.

\section{Pourriez vous me parler un peu de Productions SuperMusique?}

Ici tu es dans les bureaux de Productions SuperMusique où nous faisons de la production de concert. On a fondé notre compagnie en 1979 pour s'occuper d'un groupe de musique qu'on avait à ce moment-là et qui s'appelait Wondeur Brass. C'est un groupe de neuf femmes musiciennes qui jouaient des cuivres et moi au piano. Au début, notre mandat était vraiment de faire en sorte quon rende la musique de femmes plus visible, puis avec le temps c'est devenu un mandat un peu plus ouvert qui incorporait toute sortes de musiques de création dans le but de les rendre plus visibles, mieux diffusées ou plus diffusées à Montréal et ailleurs.

En 1988 on a organisé un festival de musiciennes innovatrices. On avait fait venir des gens de France, des États-Unis et d'Asie pour témoigner la création des femmes, pour montrer que des femmes musiciennes il y en avait. Puisqu'il $y$ avait des femmes audacieuses et aussi parce qu'on ne voulait pas juste montrer qu'il y avait des femmes chanteuses ou pianistes. On voulait montrer qu'il y avait beaucoup d'audace, que les femmes apportaient beaucoup dans l'image moderne de ce qui se passait. Alors, suite à ce festival-là, on a fait et on continue d'organiser des évènements.

Quelles sont vos préoccupations sonores? Ça pourrait être quelque chose que vous n'aimez pas, qui vous dérange ou que vous aimiez bien.

Je suis souvent préoccupée par les choix esthétiques dans la création sonore, surtout en musique électronique. Je dirais que cest vraiment facile de prendre toutes sortes de choses, de prendre beaucoup de machines et de prendre beaucoup deffets qui sont tous disponibles alors.... Tu sais il y a des gens qui utilisent des petites machines de rien du tout, c'est du "lo-fi», qu'ils se sont tous "patentés " eux-mêmes et c'est fantastique ce que ça donne; puis il y en a d’autres qui ont des gros ordinateurs tu sais, et puis qui nont pas nécessairement des choix qui m'intéressent ou dans lesquels je retrouve de la créativité. Je suis bien préoccupée par cette chose-là parce qu'effectivement, parfois cest très difficile de faire de la musique avec des gens qui ont des univers sonores d'esthétique trop loin ou trop différents à endosser.

\section{Qu'est ce que vous utilisez pour manipuler le son?}

En concert, je travaille presqu'uniquement avec un échantillonneur Roland avec les rayons infrarouges. C'est pas gros, ça se transporte bien et les rayons infra- 
rouges font que je peux vraiment jouer avec, faire une espèce de sculpture sonore. Cela me permet d'avoir un élément visuel qui permet que les gens entrent en contact avec ce que je fais... Avec un instrument comme celui-là, la gestuelle fait que les gens voient bien ce qui change le son. Cela apporte une sorte de dynamisme dans mon rapport avec le public. Moi ce que je veux, c'est avoir un rapport entre les gens, dêtre en communication ou en interaction directe autant avec les musiciens sur la scène qu'avec les gens dans la salle.

\section{Pouvez-vous décrire votre approche à la composition?}

Son approche à la composition dépend du type de projet quelle développe. Elle peut composer pour des instruments classiques comme pour des instruments électriques et électroniques, en utilisant des approches traditionnelles et non traditionnelles.

Il y a des projets où jai écrit des consignes musicales où il y a des gens qui ont des notes précises à jouer tandis que d'autres ont seulement des effets à faire sur les instruments par rapport à la composition; ou bien jécris des pièces vraiment toutes écrites pour être dirigées par un chef. Un processus de travail que j'ai beaucoup aimé et qui nétait pas nécessairement une composition comme telle, était celui pour une élaboration du disque Parasites avec Martin Tétrault. On a travaillé en studio, avec nos volumes allumés, on cherchait des sonorités qui nous plaisaient chacun pour soi. En cherchant quelque chose qui faisait mon affaire, jentendais où Martin était rendu et tout d'un coup on arrivait à quelque chose qu'on trouvait, qui allait bien ensemble et qui se complétait bien. On arrêtait, on partait l'enregistreuse et on décidait qu'on faisait une pièce de tant de minutes avec ces univers-là et on définissait comment elle pouvait évoluer.

On parlait tantôt des moments qui ont marqué mon parcours. En 93, jétais allée au Banff Centre for the Arts avec Michel F. Côté où on montait un projet qui est devenu un disque. Au début on s'entraînait à jouer et on jouait des heures et des heures sans arrêt. J'avais l'impression de faire du jogging musical. $\AA$ un moment donné, tu te répètes parce que c'est inévitable lorsque tu joues pendant longtemps, alors il faut toujours aller un peu plus loin que la dernière fois ou à côté, et je me rappelle de cette période. C'était un moment très crucial où jétais forcée d'explorer, puis d'aller plus loin, et cette chose-là est toujours restée avec moi.

En tant que femme, comment décrivez-vous votre travail dans le domaine du son? Est-ce que cela influence votre travail?

Oui cela a une influence, moi je vois tout avec les yeux qui sont les miens, ceux d'une femme, et j'entends avec ces oreilles-là.

Est-ce que vous trouvez que le domaine du son est dominé par le masculin? Quelles sont vos pensées sur cela?

Quand j’ai commencé à faire de la musique et que j’ai formé Wondeur Brass, un groupe de 8 ou 9 femmes, j’ai toujours trouvé qu'il y avait beaucoup d'hommes en musique. C’était vraiment un geste social qui visait à percuter par rapport 
à ça. Tout le contexte social a changé depuis ce temps et puis je ne pense plus vraiment à ça, parce que, heureusement, jen vois partout des femmes musiciennes qui font de l'exploration. Quand on a fait cette réunion avec Hélène Prévost (à la SRC, Le Navire « Night » 09.05.04), c'était fantastique de voir des femmes qui vivent à Montréal dont je navais jamais entendu parler et qui travaillent en musique électronique.

Diane explique qu'il y a toujours eu beaucoup d'hommes, mais la présence et la visibilité de femmes musiciennes dans tous les genres musicaux est devenue beaucoup plus forte par rapport au passé.

Les femmes ont gagné tellement de terrain dans la possibilité dêtre partout, la possibilité d'avoir des machines puis de jouer avec, d'étudier et de travailler là-dedans, de trouver des chemins et d'inventer plein de choses. Quand jétais jeune, j'aimais écouter la musique et il m'est venu à l'esprit que je voulais être chef d'orchestre. Ma mère me disait : "Ça se peut pas une femme chef d'orchestre, concentre-toi donc sur autre chose! " J'ai repensé encore à ça il n'y pas longtemps parce que j’ai vu une femme chef d'orchestre à la télé et je me suis dit bon voilà, ce nest pas impossible! Je trouve qu'au Canada, en tous cas, au Québec certainement il y a un phénomène qui fait que la démarcation des sexes est moins grande et que cette différence sest beaucoup amoindrie avec le temps.

\section{RÉSUMÉ}

Cet entretien avec Diane Labrosse a été réalisé à Montréal en décembre 2004 dans les locaux de Productions SuperMusique, dans le cadre du projet Dedans/ Dehors du studio coordonné par Dre Andra McCartney de l'Université Concordia.

Labrosse se définit comme musicienne, même si elle considère que ce titre ne couvre pas vraiment l'ensemble des rôles assumés dans divers ensembles et projets depuis 25 ans qu'elle exerce ce métier. L’essentiel de son activité est à titre de conceptrice sonore, en particulier dans les projets pour le théâtre, les concerts « live " et les performances de musique improvisée avec d'autres musiciens, danseurs ou acteurs de théâtre. Avec son instrument de prédilection, un échantillonneur, Diane crée des ambiances sonores composées de sons inhabituels, de bruits et de textures, avec un certain penchant pour des modifications brusques de hauteurs.

\section{Abstract}

This interview with Diane Labrosse was conducted in December 2004 in the offices of Production SuperMusique within the framework of Dr. Andra McCartney's In and Out Of the Sound Studio project. Andrea Jane Cornell asked Labrosse about the experiences that brought her to choose sound as a medium for her artistic expression, and the methods that she employs to create sound.

Labrosse defines herself as a musician even though she feels that this title does not encompass all the roles she fulfills in the various ensembles and projects that 
she has been involved with during the twenty five year span of her career. The majority of her sound work is in live improvisation in a concert setting, with other sound artists, musicians, dancers and theatre performers. Armed with a sampler, Diane creates sonic ambiances with unconventional sounds, noises and textures, with a penchant for drastic changes in pitch. 